Siberian Mathematical Journal, Vol. 54, No. 1, pp. 124-136, 2013

Original Russian Text Copyright (c) 2013 Oliynyk B.V. and Sushchanskǐ V.I.

\title{
THE ISOMETRY GROUPS OF THE HAMMING SPACES OF PERIODIC SEQUENCES
}

\begin{abstract}
We consider the Hamming space of periodic $(0,1)$-sequences and a continual family of its subspaces defined as direct limits of finite Hamming spaces. These subspaces form a complete lattice under inclusion which is isomorphic to the lattice of supernatural numbers. We explicitly describe the isometry groups of these spaces. This involves certain constructions similar to the hyperoctahedral groups but accounting for additional structures on the underlying sets.
\end{abstract}

DOI: $10.1134 /$ S0037446613010163

Keywords: Hamming space, Besicovitch space, isometry group, semidirect product, rooted tree, Bernoulli measure

\section{Introduction}

The Hamming spaces are among the commonest examples of finite metric spaces. Their isometry groups, the hyperoctahedral groups, characterize one of the several main symmetry types of finite structures. The normalized Hamming metric naturally extends to the set of all infinite $(0,1)$-sequences, but then it becomes a pseudometric. We obtain a metric space upon passing to the quotient by the equivalence that identifies all sequences at a vanishing distance from each other. This space, called the Besicovitch space or the Besicovitch-Hamming space [1,2], is used since the 1960s in dynamical systems and ergodic theory. Here we should first of all mention the work of Vershik, who developed a theory of decreasing sequences of measurable partitions of measure spaces [2-4] (also see [5,6]). A key moment of this theory is the development of special combinatorics of partitions; in particular, the construction of a hierarchy of partitions and groups acting on them. This framework is quite general and also encompasses various limit constructions of spaces and the related transformation groups: inductive and projective limits; the groups acting on infinite rooted trees, and so on. Vershik's hierarchy includes, for instance, the Cameron-Tarzi construction [7] which deals with the inductive limit of finite normalized Hamming spaces with the connecting embeddings determined by doubling the coordinates of $(0,1)$-vectors. The limit space is naturally interpreted as the space of finite unions of half-open subintervals of the interval $[0,1)$ with binary-rational endpoints; furthermore, the distance between two unions of this type equals the sum of lengths of the segments constituting their symmetric difference. This representation has made it possible to study the isometry groups of both the Cameron-Tarzi space itself and its completion.

The normalized Hamming metric extends to the set of periodic $(0,1)$-sequences once we define the distance between two sequences as the normalized distance between their beginnings whose lengths are divisible by the periods of these sequences. The arising metric space isometrically embeds into the Besicovitch-Hamming space, and since the latter is complete, the completion of the space of periodic $(0,1)$-sequences coincides with its closure in this ambient space.

The Hamming space of periodic $(0,1)$-sequences includes a continual family of subspaces defined as direct limits of finite Hamming spaces. These subspaces are naturally parametrized by supernatural numbers and form a lattice under inclusion which is isomorphic to the lattice of supernatural numbers under divisibility. The closure of each of these infinite spaces coincides with the closure of the space of all periodic $(0,1)$-sequences. The goal of this article is to study the isometry group of the spaces of the family. This involves group constructions quite similar to the construction of hyperoctahedral groups,

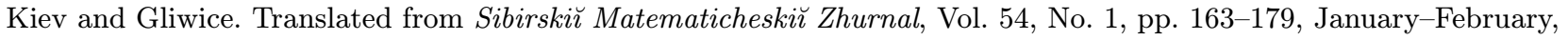
2013. Original article submitted October 23, 2012. 
but accounting for additional structures on the underlying sets.

Let us describe the structure of this article. In Section 2 we define the Hamming space of all periodic $(0,1)$-sequences, select a family of its subspaces parametrized by supernatural numbers, and give an independent description of them as inductive limits of finite Hamming spaces.

In Section 3 we describe embeddings of the Hamming spaces of $u$-periodic sequences (where $u$ runs over the set of infinite supernatural numbers) into the Besicovitch-Hamming space and their realization on the boundaries of spherically homogeneous rooted trees (Theorem 1). For the reader's convenience we include the detailed proofs of all statements even though some of them are of a folklore character.

In Section 4 we describe an explicit construction of isometry groups of the Hamming space of $u$ periodic sequences (Theorem 2), which is quite similar to the construction of hyperoctahedral groups, but accounts for the fact that the boundary of a spherically homogeneous rooted tree is an $m m$-space, that is, a metric space equipped with a Bernoulli measure. Moreover, we show in Theorem 3 how to construct this isometry group from finite hyperoctahedral groups.

The notation of this article is standard. The authors take this chance to thank A. M. Vershik, Ya. V. Lavrenyuk, and V. V. Nekrashevich for useful discussions.

\section{The Hamming Space of Periodic $(0,1)$-Sequences}

2.1. Fix a positive integer $n$. Recall that the Hamming metric space $H_{n}$ is the set of all $(0,1)$ sequences of length $n$ endowed with the metric $d_{H_{n}}$ defined as

$$
d_{H_{n}}(\bar{x}, \bar{y})=\sum_{i=1}^{n}\left|x_{i}-y_{i}\right|,
$$

where $\bar{x}=\left(x_{1}, \ldots, x_{n}\right), \bar{y}=\left(y_{1}, \ldots, y_{n}\right) \in H_{n}$. The normalized Hamming space $\hat{H}_{n}$ is the space defined on the same set endowed with the normalized metric $\hat{d}_{H_{n}}=\frac{1}{n} d_{H_{n}}$.

An infinite $(0,1)$-sequence $\mathbf{a}=\left(a_{1}, a_{2}, \ldots\right)$ is called periodic whenever there exists a positive integer $m$ such that $a_{i}=a_{i+m}$ for all $i \in \mathbb{N}$. Every number $m$ with this property is called a period of $\mathbf{a}$. Denote the set of all periodic $(0,1)$-sequences by $\mathscr{H}$. The normalized metric $\hat{d}_{H_{n}}$, defined above for $n \in \mathbb{N}$, extends naturally to $\mathscr{H}$. Namely, given two sequences $\mathbf{x}=\left(x_{1}, x_{2}, \ldots\right)$ and $\mathbf{y}=\left(y_{1}, y_{2}, \ldots\right)$ in $\mathscr{H}$ with periods $m$ and $n$, denote their common period by $l$ and put

$$
d_{\mathscr{H}}(\mathbf{x}, \mathbf{y})=\frac{1}{l} \sum_{i=1}^{l}\left|x_{i}-y_{i}\right| .
$$

The right-hand side of (2) is clearly independent of the choice of $m, n$, and $l$; therefore, we can choose $l$ equal, for instance, to the least common multiple of $m$ and $n$ or to their product $m n$. Thus, $d_{\mathscr{H}}$ is well-defined.

Definition 1. The metric space $\left(\mathscr{H}, d_{\mathscr{H}}\right)$ is called the Hamming space of periodic $(0,1)$-sequences.

2.2. A supernatural number (or Steinitz number) is a formal expression of the form $\prod_{p \in \mathbb{P}} p^{k_{p}}$, where $\mathbb{P}$ stands for the set of all primes, while $k_{p} \in \mathbb{N} \cup\{0, \infty\}$ for every $p \in \mathbb{P}$. Denote the set of all supernatural numbers by $\mathbb{S N}$. This set admits a divisibility relation $\mid$ : a supernatural number $u=\prod_{p \in \mathbb{P}} p^{k_{p}}$ divides a supernatural number $v=\prod_{p \in \mathbb{P}} p^{n_{p}}$ whenever $k_{p} \leq n_{p}$ for all $p \in \mathbb{P}$. Furthermore, the symbol $\infty$ is greater than all positive integers and zero. The relation $\mid$ is a partial order on $\mathbb{S N}$, and the poset $(\mathbb{S N}, \mid)$ is a complete lattice with top $\left(I=\prod_{p \in \mathbb{P}} p^{\infty}\right)$ and bottom $\left(1=\prod_{p \in \mathbb{P}} p^{0}\right)$. The lattice $(\mathbb{S N}, \mid)$ is the completion of $(\mathbb{N}, \mid)$, and the lattice operations $\vee$ and $\wedge$ in $\mathbb{S N}$ are the straightforward generalizations of the corresponding operations of $\mathbb{N}$. The elements of $\mathbb{S N} \backslash \mathbb{N}$ are called infinite supernatural numbers.

In the Hamming space of all periodic sequences we select a family of subspaces parametrized by supernatural numbers. Given a supernatural number $u \in \mathbb{S N}$, refer to a sequence a as u-periodic whenever its minimal period divides $u$. Refer to the subspace $\mathscr{H}(u)$ of all $u$-periodic sequences in $\mathscr{H}$ as the Hamming space of all u-periodic $(0,1)$-sequences. Observe the following easy properties of these spaces. 
Lemma 1. (1) If $u$ is a natural number then $\mathscr{H}(u)$ is isometric to the normalized Hamming space $\widehat{H}_{u}$. (2) $\mathscr{H}(I)=\mathscr{H}$.

(3) $\mathscr{H}(u) \subseteq \mathscr{H}(v)$ if and only if $u \mid v$.

(4) For every supernatural number $u$ the set $\operatorname{Dist}\left(\mathscr{H}(u), d_{\mathscr{H}}\right)$ of values of $d_{\mathscr{H}}$ on $\mathscr{H}(u)$ is determined as

$$
\operatorname{Dist}\left(\mathscr{H}(u), d_{\mathscr{H}}\right)=\mathbb{Q}_{u} \cap[0,1]
$$

where $\mathbb{Q}_{u}$ is the set of fractions whose denominator is a divisor of $u$.

(5) If $u \neq v$ then $\mathscr{H}(u)$ and $\mathscr{H}(v)$ are not isometric.

(6) The spaces $\mathscr{H}(u)$ for $u \in \mathbb{S N}$ form a lattice under inclusion which is isomorphic to the lattice of supernatural numbers under divisibility.

2.3. We can characterize each space $\mathscr{H}(u)$ for $u \in \mathbb{S N}$ independently by using the concept of inductive limit of metric spaces. Given positive integers $k, l$, and $s$ with $k \mid l$ and $l=k \cdot s$, define the embedding $f_{s}$ of $\widehat{H}_{k}$ into $\widehat{H}_{l}$ by putting, for an arbitrary vector $\bar{x}=\left(x_{1}, \ldots, x_{k}\right) \in \widehat{H}_{k}$,

$$
f_{s}\left(x_{1}, \ldots, x_{k}\right)=(\underbrace{x_{1}, \ldots, x_{k}\left|x_{1}, \ldots, x_{k}\right| \ldots \mid x_{1}, \ldots, x_{k}}_{k \cdot s}) .
$$

The embedding $f_{s}$ is an isometry which we call the diagonal embedding of multiplicity $s$.

Take now a strictly increasing divisible sequence $\tau=\left(m_{1}, m_{2}, \ldots\right)$ of positive integers, that is, $m_{i} \mid m_{i+1}$ for all $i \in \mathbb{N}$. The sequence $\tau$ determines the direct system of normalized Hamming spaces

$$
\Sigma(\tau)=\left\langle\widehat{H}_{m_{i}}, f_{s_{i}}\right\rangle_{i \in \mathbb{N}}
$$

with the diagonal embeddings $f_{s_{i}}$ of multiplicities $s_{i}=\frac{m_{i}}{m_{i-1}}$, where $i>2$, determined by (3). We can identify the limit space

$$
\mathscr{H}(\tau)=\underline{\varliminf}\left\langle\widehat{H}_{m_{i}}, f_{s_{i}}\right\rangle
$$

of the system (4) with the space of periodic sequences whose periods are divisors of the terms of $\tau$. Refer to the supernatural number arising when we consider the infinite product

$$
m_{1} \cdot \frac{m_{2}}{m_{1}} \cdot \frac{m_{3}}{m_{2}} \cdot \frac{m_{4}}{m_{3}} \cdot \ldots
$$

as the characteristic sequence of $\tau$ and denote it by $\operatorname{char}(\tau)$. A positive integer is a divisor of the terms of $\tau$ if and only if it is a divisor of the supernatural number $\operatorname{char}(\tau)$. Observe also that we can naturally regard $\mathscr{H}(\tau)$ as a subspace of $\mathscr{H}$. This directly implies

Lemma 2. For every strictly increasing divisible sequence $\tau$ the space $\mathscr{H}(\tau)$ regarded as a subspace of $\mathscr{H}$ coincides with $\mathscr{H}(u)$, where $u=\operatorname{char}(\tau)$.

Refer to $\mathscr{H}(\tau)$ as the Hamming space of all u-periodic $(0,1)$-sequences. Lemmas 1,2 , and formula $(5)$ directly imply

Lemma 3. The spaces $\mathscr{H}(\tau)$ and $\mathscr{H}(\varsigma)$ determined by two divisible sequences $\tau$ and $\varsigma$ are isometric if and only if $\operatorname{char}(\tau)=\operatorname{char}(\varsigma)$.

Thus, the family of pairwise nonisometric spaces of the form $\mathscr{H}(\tau)$, where $\tau$ is some strictly increasing divisible sequence, is uniquely parametrized by supernatural numbers. In fact, we can define this parametrization for a larger class of inductive limits of finite Hamming spaces. These limits are determined by the types of connecting embeddings, which are constructed from partitions of the sets of indices. More exactly, take two positive integers $k$ and $s$ and consider a partition $\pi=\left\langle R_{1}, R_{2}, \ldots, R_{k}\right\rangle$ of the set of indices $\{1,2, \ldots, k s\}$ into $k$ cardinality $s$ subsets.

Definition 2. Refer as a $\pi$-embedding of the Hamming space $\widehat{H}_{k}$ into the space $\widehat{H}_{k s}$ to the mapping $f_{\pi}: \widehat{H}_{k} \rightarrow \widehat{H}_{k s}$ determined for every $\bar{x}=\left(x_{1}, x_{2}, \ldots, x_{k}\right)$ by the condition

$$
f_{\pi}\left(x_{1}, x_{2}, \ldots, x_{k}\right)=\left(y_{1}, y_{2}, \ldots, y_{k s}\right),
$$

where for all $i \in R_{j}$ the coordinates $y_{i}$ are equal to $x_{j}$ (for $1 \leq j \leq k$ ). 
Each $\pi$-embedding is an isometric embedding of the normalized Hamming space $\widehat{H}_{k}$ into $\widehat{H}_{k s}$. Take a strictly increasing divisible sequence $\tau=\left(m_{1}, m_{2}, \ldots\right)$ of positive integers with $m_{i+1}=m_{i} s_{i+1}$ and a partition $\pi_{i}$ of the set of indices $\left\{1,2, \ldots, m_{i+1}\right\}$ into $s_{i+1}$-element subsets. These data uniquely determine the direct spectrum $\left\langle\widehat{H}_{m_{i}}, f_{\pi_{i}}\right\rangle_{i \in \mathbb{N}}$ of Hamming spaces, that is, we can consider its limit space.

Lemma 4. For every sequence $\pi_{i}, i \in \mathbb{N}$, of partitions the limit space $\underline{\lim }\left\langle\widehat{H}_{m_{i}}, f_{\pi_{i}}\right\rangle$ is isometric to $\mathscr{H}(\tau)$.

Proof. We can obtain the embedding $f_{\pi}$ of $\widehat{H}_{k}$ into $\widehat{H}_{k s}$ which is determined by a partition $\pi$ of the set $\{1,2, \ldots, k s\}$ of indices from the diagonal embedding $f_{s}: \widehat{H}_{k} \rightarrow \widehat{H}_{k s}$ by a fixed permutation of coordinates of the vectors $\nu$. Suppose that $\psi_{k, s}: \widehat{H}_{k s} \rightarrow \widehat{H}_{k s}$ is a transformation of $\widehat{H}_{k s}$ determined by this permutation:

$$
\psi_{k, s}\left(x_{1}, x_{2}, \ldots, x_{k s}\right)=\left(x_{\nu(1)}, x_{\nu(2)}, \ldots, x_{\nu(k s)}\right) .
$$

The transformation $\psi_{k, s}$ is an isometry of $\widehat{H}_{k s}$; furthermore, the diagram

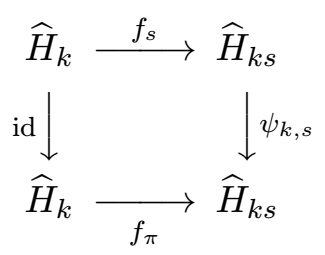

commutes (here id stands for the identity transformation). Therefore, the infinite diagram

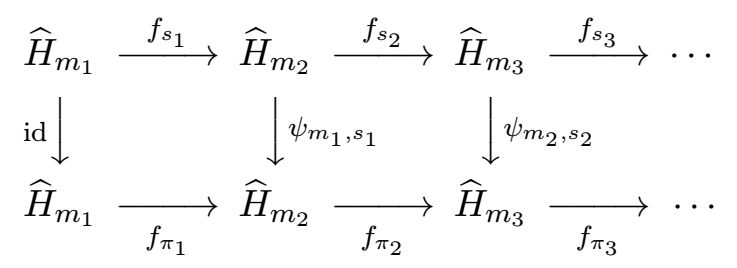

constructed from the direct spectra $\left\langle\widehat{H}_{m_{i}}, f_{s_{i}}\right\rangle_{i \in \mathbb{N}}$ and $\left\langle\widehat{H}_{m_{i}}, f_{\pi_{i}}\right\rangle_{i \in \mathbb{N}}$ also commutes. Since all connecting morphisms $\psi_{m_{i}, s_{i}}$ for $i \in \mathbb{N}$ are isometries, the limit spaces of these direct spectra are isometric.

EXAMPLE 1. Refer as doubling to the mapping $\varphi_{k}: \widehat{H}_{k} \rightarrow \widehat{H}_{2 k}$ determined as

$$
\varphi_{k}\left(x_{1}, x_{2}, \ldots, x_{k}\right)=\left(x_{1}, x_{1}, x_{2}, x_{2}, \ldots, x_{k}, x_{k}\right) .
$$

The doubling $\varphi_{k}$ is a $\pi_{k}$-embedding for $s=2$ and the partition

$$
\pi_{k}=\{\{1,2\},\{3,4\}, \ldots,\{2 k-1,2 k\}\}
$$

of the set $\{1, \ldots, 2 k\}$ of indices. The direct spectrum $\Phi=\left\langle\widehat{H}_{2^{l}}, \varphi_{2^{l}}\right\rangle_{l \in \mathbb{N}}$ of normalized Hamming spaces determines a countable metric space, studied in [7], as an interesting generalization of finite Hamming spaces to the infinite-dimensional case.

\section{Isometric Embeddings of the Hamming Spaces of Periodic $(0,1)$-Sequences}

3.1. An embedding into the Besicovitch space. Denote by $\{0,1\}^{\mathbb{N}}$ the space of all (right-)infinite $(0,1)$-sequences. Endow it with a distance function by putting

$$
\hat{d}_{B}(\mathbf{x}, \mathbf{y})=\limsup _{n \rightarrow \infty} \hat{d}_{H_{n}}\left(\left(x_{1}, \ldots, x_{n}\right),\left(y_{1}, \ldots, y_{n}\right)\right)
$$

for all sequences $\mathbf{x}=\left(x_{1}, x_{2}, \ldots\right)$ and $\mathbf{y}=\left(y_{1}, y_{2}, \ldots\right)$. 
The mapping $\hat{d}_{B}$ thus defined is a pseudometric, that is, there exist distinct sequences $\mathbf{x}$ and $\mathbf{y}$ with $\hat{d}_{B}(\mathbf{x}, \mathbf{y})=0$. The pseudometric (6) defines the equivalence $\sim_{\hat{d}_{B}}$ on $\{0,1\}^{\mathbb{N}}$ with $\mathbf{x} \sim_{\hat{d}_{B}} \mathbf{y}$ if and only if $\hat{d}_{B}(\mathbf{x}, \mathbf{y})=0$. Denote by $\mathscr{X}_{B}=\{0,1\}^{\mathbb{N}} / \sim_{\hat{d}_{B}}$ the quotient set by this equivalence. The function $\hat{d}_{B}$ induces the metric $d_{B}$ on $\mathscr{X}_{B}$, and the metric space $\left(\mathscr{X}_{B}, d_{B}\right)$ is called the Besicovitch space or the Besicovitch-Hamming space (see $[1,2]$ for instance). The passage from $\{0,1\}^{\mathbb{N}}$ to $\mathscr{X}_{B}$ amounts to gluing all sequences the distance between which vanishes. In particular, all almost zero sequences (and not only those) are glued into a singleton. The Besicovitch space enjoys the following properties.

Lemma 5 [1]. The metric space $\left(\mathscr{X}_{B}, d_{B}\right)$ is complete, nonseparable, and not locally compact.

Denote by $[\mathbf{x}]$ the coset of $\sim_{\hat{d}_{B}}$ which includes $\mathbf{x} \in\{0,1\}^{\mathbb{N}}$.

It is not difficult to verify

Proposition 1. The mapping $h: \mathbf{x} \rightarrow[\mathbf{x}]$ is an isometric embedding of the space $\mathscr{H}$ of periodic $(0,1)$-sequences into the Besicovitch space $\mathscr{X}_{B}$.

Since $\left(\mathscr{X}_{B}, d_{B}\right)$ is a complete space, the completion $\mathscr{C} \mathscr{H}$ of the space $\mathscr{H}$ of all periodic $(0,1)$ sequences is isometric to the closure $\mathscr{W}$ of the image of $\mathscr{H}$ under $h$. The space $\mathscr{H}$ is obviously invariant under shift, that is, the shift

$$
\sigma: \mathscr{X}_{B} \rightarrow \mathscr{X}_{B}, \quad \sigma\left(x_{1}, x_{2}, x_{3}, \ldots\right)=\left(x_{2}, x_{3}, \ldots\right) .
$$

Therefore, the subspace $\mathscr{W} \subset \mathscr{X}_{B}$ is also invariant under shift, and since $\mathscr{W}$ is compact, $\mathscr{W}$ is a natural range for dynamical systems on $(0,1)$-sequences.

Observe that for every infinite supernatural number $u$ the image of the subspace $\mathscr{H}(u)$ of $\mathscr{H}$ under $h$ is also invariant under shift, and the closures of all these spaces coincide with $\mathscr{W}$.

3.2. Representation of periodic Hamming spaces on the boundaries of spherically homogeneous rooted trees. Consider a locally finite rooted tree $T$, that is, a tree with a vertex designated as the root in which finitely many edges emanate from each vertex.

It is natural to divide the set of vertices of a tree $T$ with root $v_{0}$ into levels (spheres): level $n$, denoted by $L_{n}$, consists of the vertices connected to the root by a path of length $n \geq 0$.

A rooted tree $\left(T, v_{0}\right)$ is called spherically homogeneous whenever the degrees of all vertices on the same level are equal. Up to an isomorphism of rooted trees, this tree is uniquely determined by its spherical index (or branching index), which is a sequence of positive integers $\left[s_{1} ; s_{2} ; \ldots,\right]$ such that $s_{1}$ is the degree of the root $v_{0}$ and $s_{i}+1$ for all $i>1$ is the degree of vertices on level $L_{i-1}$. If a tree $\left(T, v_{0}\right)$ has spherical index $\left[s_{1} ; s_{2} ; \ldots,\right]$ then the sequence of cardinalities $m_{k}=\left|L_{k}\right|$, for $k \geq 1$, of its levels is divisible since $m_{k}=s_{1} \cdot s_{2} \cdot \ldots \cdot s_{k}$.

The boundary $\partial T$ of a tree $T$ is the set of infinite paths without repetitions starting at the root $v_{0}$. (These paths are called the ends of $T$.) The boundary $\partial T$ carries the structure of a metric space; namely, the distance between two given paths $\gamma_{1}, \gamma_{2} \in \partial T$ is defined as

$$
\rho\left(\gamma_{1}, \gamma_{2}\right)= \begin{cases}\frac{1}{k+1} & \text { if } \gamma_{1} \neq \gamma_{2}, \\ 0 & \text { if } \gamma_{1}=\gamma_{2},\end{cases}
$$

where $k$ is the index of the level on which the paths $\gamma_{1}$ and $\gamma_{2}$ diverge. The space $(\partial T, \rho)$ is an ultrametric and totally disconnected compact set of diameter 1 .

Given a strictly increasing divisible sequence $\tau=\left(m_{1}, m_{2}, \ldots\right)$, consider the sequence $\left[s_{1} ; s_{2} ; \ldots,\right]$ of its ratios, that is,

$$
s_{1}=m_{1}, \quad s_{i}=\frac{m_{i}}{m_{i-1}}, \quad i \geq 2 .
$$

Denote by $T_{\tau}$ the spherically homogeneous rooted tree with spherical index $\left[s_{1} ; s_{2} ; \ldots,\right]$, and by $\rho_{\tau}$, the metric (7) on $\partial T_{\tau}$. The automorphism group of $T_{\tau}$ acts on it spherically transitively, which means that 
all levels of $T_{\tau}$ are preserved and the action on each level is transitive. Furthermore, the group Aut $T_{\tau}$ is isomorphic to the infinitely iterated wreath product of symmetric groups of degrees $s_{1}, s_{2}, \ldots$ :

$$
\text { Aut } T_{\tau} \simeq l_{i=1}^{\infty} S_{s_{i}}
$$

(see [8] for instance). Each automorphism $\alpha$ of $T_{\tau}$ acts as an isometry on the boundary $\left(\partial T_{\tau}, \rho_{\tau}\right)$ and conversely:

$$
\operatorname{Iso}\left(\partial T_{\tau}, \rho_{\tau}\right)=\operatorname{Aut} T_{\tau}
$$

The action of Aut $T_{\tau}$ on $\partial T_{\tau}$ is transitive. For $\tau_{1} \neq \tau_{2}$ the spaces $\partial T_{\tau_{1}}$ and $\partial T_{\tau_{2}}$ are not isometric, and their isometry groups are not isomorphic.

Given a vertex $v$ of the rooted tree $T_{\tau}$, refer as the cylindrical set $C_{v}$ corresponding to $v$ to the set of all ends of $T_{\tau}$ passing through $v$ :

$$
C_{v}=\left\{\gamma \in \partial T_{\tau} \mid v \in \gamma\right\} .
$$

The metric $\rho_{\tau}$ induces some topology on $\partial T_{\tau}$. The clopen subsets are precisely finite unions of cylindrical sets.

Introduce a Borel probability measure on the $\sigma$-algebra of clopen sets of $\partial T_{\tau}$ : the Bernoulli measure $\mu$ defined as

$$
\mu\left(C_{v}\right)=\frac{1}{n_{v}},
$$

where $n_{v}$ is the number of vertices of $T_{\tau}$ on the level containing $v$. It is a unique probability measure on $\partial T_{\tau}$ invariant under the action of the isometry group $\operatorname{Iso}\left(\partial T_{\tau}, \rho_{\tau}\right)$. In addition, $\left(\partial T_{\tau}, \mu\right)$ is isomorphic as a measure space to $([0,1], l)$, where $l$ is the Lebesgue measure (see [8] for instance).

Using the measure $\mu$ on the set $\Omega T_{\tau}$ of all clopen subsets of $\partial T_{\tau}$, define the metric $d_{\mu}$ by putting

$$
d_{\mu}(A, B)=\mu(A \triangle B)
$$

for all clopen sets $A$ and $B$ of $\partial T_{\tau}$. The definition of $\mu$ implies that only the empty subset can have measure zero; therefore, the metric $d_{\mu}$ is well-defined.

Theorem 1. Given a strictly increasing divisible sequence $\tau=\left(m_{1}, m_{2}, \ldots\right)$ of positive integers with the sequence $\left[s_{1} ; s_{2} ; \ldots,\right]$ of its ratios (8), consider the spherically homogeneous rooted tree $T_{\tau}$ of spherical index $\left[s_{1} ; s_{2} ; \ldots,\right]$ and a supernatural number $u$ with $\operatorname{char}(\tau)=u$. Then the Hamming space $\mathscr{H}(u)$ of all $u$-periodic $(0,1)$-sequences is isometric to the space $\Omega T_{\tau}$ of all clopen subsets of $\partial T_{\tau}$ equipped with the metric $d_{\mu}$ defined by (10).

Proof. Denote by $\mathscr{C}_{k}$ the set of all possible unions of cylindrical sets $C_{v}$ determined by the vertices of $T_{\tau}$ on level $k \geq 1$, regarded as a metric space with the metric (10). Since $C_{v_{1}} \neq C_{v_{2}}$ for $v_{1} \neq v_{2}$, the sets $C_{v}$ for $v \in L_{k}$ partition $\partial T_{\tau}$ into $m_{k}$ subsets. Therefore, $\left|\mathscr{C}_{k}\right|=2^{m_{k}}$. Enumerate the vertices in $L_{k}$, that is, write $L_{k}=\left\{v_{1}, \ldots, v_{m_{k}}\right\}$.

If $A=\bigcup_{i \in I_{A}} C_{v_{i}}$ and $B=\bigcup_{j \in I_{B}} C_{v_{j}}$, where $I_{A}, I_{B} \subseteq\left\{1, \ldots, m_{k}\right\}$ then

$$
A \triangle B=\bigcup_{i \in I_{A}} \bigcup_{i \in I_{B}}\left(C_{v_{i}} \triangle C_{v_{j}}\right)
$$

But the symmetric difference on the right-hand side satisfies

$$
C_{v_{i}} \triangle C_{v_{j}}= \begin{cases}\varnothing & \text { if } i=j, \\ C_{v_{i}} \cup C_{v_{j}} & \text { if } i \neq j .\end{cases}
$$

Therefore, $A \triangle B$ is the union of cylindrical sets determined by the vertices in $L_{k}$. Furthermore, the definition of $\mu$ yields

$$
\mu(A \triangle B)=\frac{\left|I_{A} \triangle I_{B}\right|}{m_{k}} .
$$


Associated to each set $Y$ in $\mathscr{C}_{k}$ there is a unique characteristic vector $\chi(Y)$. This is the $(0,1)$-vector of length $m_{k}$ whose coordinate $l$ equals 1 if and only if $C_{v_{l}} \subseteq Y$. The mapping $\chi: \mathscr{C}_{k} \rightarrow \widehat{H}_{m_{k}}$ is a bijection and

$$
d_{\mu}(A, B)=\mu(A \triangle B)=\frac{\left|I_{A} \triangle I_{B}\right|}{m_{k}}=\hat{d}_{H_{m_{k}}}(\chi(A), \chi(B))
$$

for all $A, B \in \mathscr{C}_{k}$. Consequently, $\left(\mathscr{C}_{k}, d_{\mu}\right)$ is isometric to the normalized Hamming space $\widehat{H}_{m_{k}}$.

Every level $k$ cylindrical set is subdivided into $s_{k+1}$ cylindrical level $(k+1)$ subsets of $T_{\tau}$. This determines an injection $\varpi_{k}$ of $\mathscr{C}_{k}$ into $\mathscr{C}_{k+1}$ so that the diagram

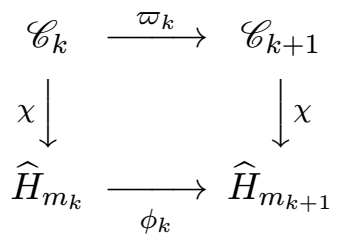

commutes, where $\varphi_{k}: \widehat{H}_{m_{k}} \rightarrow \widehat{H}_{m_{k+1}}$ is a repetition of multiplicity $s_{k+1}$, which means

$$
\varphi_{k}\left(x_{1}, \ldots, x_{m_{k}}\right)=(\underbrace{x_{1}, \ldots, x_{1}}_{s_{k+1}}, \ldots, \underbrace{x_{m_{k}}, \ldots, x_{m_{k}}}_{s_{k+1}}) .
$$

This implies that the union of the increasing chain $\mathscr{C}_{1} \subset \mathscr{C}_{2} \subset \mathscr{C}_{3} \ldots$ of spaces, which is the space of all clopen subsets of $\partial T_{\tau}$ equipped with the metric $d_{\mu}$, is isometric to the direct limit of the spaces $\widehat{H}_{m_{1}} \hookrightarrow \widehat{H}_{m_{2}} \hookrightarrow \cdots$, which, in turn, is isometric by Lemma 4 to the Hamming space $\mathscr{H}(\tau)$ of all $u$-periodic $(0,1)$-sequences.

The proof of the theorem is complete.

Corollary 1. The completion of $\mathscr{H}(\tau)$ is isometric to the space of all measurable subsets (up to measure zero sets) of $\left(\partial T_{\tau}, \mu\right)$ equipped with the metric $d_{\mu}$ defined in (10).

The space $\left(\partial T_{\tau}, \mu\right)$ is a standard probability space. Therefore, the completion of $\mathscr{H}(\tau)$ is independent of the choice of a divisible sequence $\tau=\left(m_{1}, m_{2}, \ldots\right)$. Thus, we have

Corollary 2. For every infinite strictly increasing divisible sequence $\tau$ the completion of the space $\mathscr{H}(\tau)$ is isometric to the completion $\mathscr{C} \mathscr{H}$ of the space $\mathscr{H}$ of all periodic $(0,1)$-sequences.

\section{The Isometry Group of the Hamming Space $H(u)$}

4.1. The isometry group Iso $H_{n}$ of the Hamming space $H_{n}$ is isomorphic (see [9] for instance) to the wreath product $Z_{2} 2 S_{n}$ of the symmetric group of degree $n$ and the cyclic group $Z_{2}$ acting regularly on $\{0,1\}$. In other words, the elements of this group are the pairs of the form $[\alpha, g]$ with $\alpha \in S_{n}$ and a mapping $g:\{1,2, \ldots, n\} \rightarrow Z_{2}$; furthermore, such a pair acts on a $(0,1)$-vector $\bar{x}=\left(x_{1}, \ldots, x_{n}\right) \in H_{n}$ as

$$
x^{[\alpha, g]}=\left(x_{1^{\alpha}}^{g(1)}, x_{2^{\alpha}}^{g(2)}, \ldots, x_{n^{\alpha}}^{g(n)}\right) .
$$

The group Iso $H_{n}$ of permutations is the automorphism group of the $n$-dimensional cube and the $n$-dimensional octahedron (cross-polytope); therefore, it is called the ( $n$-dimensional) hyperoctahedral group. We can consider a similar construction for symmetric groups over arbitrary sets: if $S(Y)$ is the symmetric group over a set $Y$ then $Z_{2} \prec S(Y)$ is the group whose elements are the pairs of the form $[\alpha, g]$ with $\alpha \in S(Y)$ and $g \in Z_{2}^{Y}$; furthermore, the action of these pairs on the elements of the set $\{0,1\}^{Y}$ is defined as

$$
h(t)^{[\alpha, g]}=\left[h\left(t^{\alpha}\right)\right]^{g(t)}, \quad h(t) \in\{0,1\}^{Y} .
$$

This yields the group $\left(Z_{2} \succ S(Y),\{0,1\}^{Y}\right)$ of permutations, which we may regard as a generalization of the hyperoctahedral groups of finite dimension to the infinite-dimensional case. But the group thus 
defined is too large for our purposes. We are interested in the cases when $Y$ carries the structure of a topological (in particular, metric) space or a measure space, and the construction is defined while taking this additional structure into account.

(i) Given a topological space $Y$, consider the group of homeomorphisms $\operatorname{Homeo}(Y)$ of $Y$ and the group $\operatorname{Fun}\left(Y, Z_{2}\right)$ of all mappings from $Y$ into $Z_{2}$ with the componentwise addition $\oplus$ modulo 2:

$$
\left(h_{1} \oplus h_{2}\right)(x)=h_{1}(x) \oplus h_{2}(x), \quad x \in Y,
$$

for all $h_{1}, h_{2} \in \operatorname{Fun}\left(Y, Z_{2}\right)$. Denote by $\operatorname{Fun}_{C}\left(Y, Z_{2}\right)$ the $\operatorname{subgroup} \operatorname{Fun}\left(Y, Z_{2}\right)$ consisting of all continuous mappings from $Y$ into $Z_{2}$. The group $\operatorname{Homeo}(Y)$ acts on $\operatorname{Fun}_{C}\left(Y, Z_{2}\right)$ by generalized translations: given $g \in \operatorname{Homeo}(Y)$ and $h \in \operatorname{Fun}_{C}\left(Y, Z_{2}\right)$, put

$$
h^{g}(x)=h\left(x^{g}\right), \quad x \in Y .
$$

This action is an automorphism of $\operatorname{Fun}_{C}\left(Y, Z_{2}\right)$. Consequently, we can consider the semidirect product

$$
\operatorname{Fun}_{C}\left(Y, Z_{2}\right) \rtimes \operatorname{Homeo}(Y)
$$

and regard it as the topological analog of the wreath product $Z_{2}$ \ $S(Y)$.

(ii) Given a measure space $(Y, \mathscr{F}, \mu)$, consider the group $\operatorname{Fun}_{\mu}\left(Y, Z_{2}\right)$ of measurable mappings from $Y$ into $Z_{2}$ with the group operation (12). The group $\operatorname{Aut}(Y, \mu)$ acts on $\operatorname{Fun}_{\mu}\left(Y, Z_{2}\right)$ by automorphisms in accordance with (13); therefore, the semidirect product

$$
\operatorname{Fun}_{\mu}\left(Y, Z_{2}\right) \rtimes \operatorname{Aut}(Y, \mu)
$$

arises naturally, which we may regard as the measurable analog of the wreath product $Z_{2} \prec S(Y)$.

(iii) Given a space $(Y, \rho, \mu)$ with a metric $\rho$ and a measure $\mu$, define $\operatorname{Aut}_{C}(Y, \mu)$ as the intersection of $\operatorname{Homeo}_{(}(Y)$ and $\operatorname{Aut}(Y, \mu)$. Since all continuous functions in $\operatorname{Fun}\left(Y, Z_{2}\right)$ are measurable, $\operatorname{Aut}_{C}(Y, \mu)$ acts by automorphisms on $\operatorname{Fun}_{C}\left(Y, Z_{2}\right)$. This means that we can construct the semidirect product

$$
\operatorname{Fun}_{C}\left(Y, Z_{2}\right) \rtimes \operatorname{Aut}_{C}(Y, \mu),
$$

which is a continuously measurable analog of the wreath product $Z_{2} \prec S(Y)$.

The semidirect products (14)-(16) act on the sets $\operatorname{Fun}_{C}(Y,\{0,1\})$ and $\operatorname{Fun}_{\mu}(Y,\{0,1\})$ in accordance with (11) and (13). This yields a transformation group which we naturally regard as the appropriate analog (topological, measurable, or continuously measurable) of the hyperoctahedral groups. Below we need only the semidirect products (15) and (16). In order to emphasize that these constructions are fully analogous to the wreath product, we will denote them by $Z_{2} \imath_{\mu} \operatorname{Aut}(Y, \mu)$ and $Z_{2} \imath_{C \mu} \operatorname{Aut} C(Y, \mu)$ respectively.

4.2. Let us now study the structure of the isometry groups of the Hamming space $\mathscr{H}(u)$, where $u$ is some supernatural number.

Theorem 2. Let $u$ be a supernatural number and let $\tau=\left(m_{1}, m_{2}, \ldots\right)$ be a strictly increasing divisible sequence of positive integers with $\operatorname{char}(\tau)=u$. The isometry group of the Hamming space $\mathscr{H}(u)$ is isomorphic as a transformation group to the continuously measurable analog (16) of the hyperoctahedral group:

$$
\text { Iso } \mathscr{H}(u) \simeq Z_{2} \imath_{C \mu} \operatorname{Aut}_{C}\left(\partial T_{\tau}, \mu\right)
$$

where $T_{\tau}$ is the spherically homogeneous rooted tree and $\mu$ is the Bernoulli measure (9) on the $\sigma$-algebra of clopen sets of $\partial T_{\tau}$.

In order to prove this theorem we need two auxiliary combinatorial statements concerning the Hamming spaces.

Given a pair $x, y$ of points in some metric space $(Y, \rho)$, the interval with endpoints $x$ and $y$ is the set

$$
[x, y]=\{z \in Y \mid \rho(x, z)+\rho(z, y)=\rho(x, y)\} .
$$




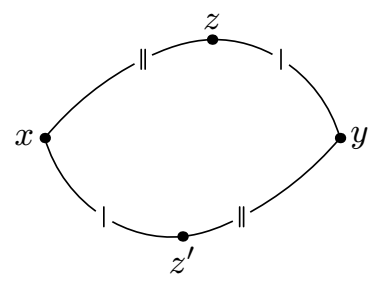

Fig. 1

Lemma 6. Given $u \in \mathbb{S N}$, consider the space $\mathscr{H}(u)$ of $u$-periodic $(0,1)$-sequences. For all $\mathbf{x}, \mathbf{y}, \mathbf{z}$ with $\mathbf{z} \in[\mathbf{x}, \mathbf{y}]$ there exists a unique point $\mathbf{z}_{\mathbf{1}} \in[\mathbf{x}, \mathbf{y}]$ with (Fig. 1)

$$
\begin{aligned}
d_{\mathscr{H}}\left(\mathbf{z}, \mathbf{z}_{\mathbf{1}}\right)= & d_{\mathscr{H}}(\mathbf{x}, \mathbf{y}), \quad d_{\mathscr{H}}\left(\mathbf{y}, \mathbf{z}_{\mathbf{1}}\right)=d_{\mathscr{H}}\left(\mathbf{x}, \mathbf{z}_{\mathbf{1}}\right) \\
& d_{\mathscr{H}}\left(\mathbf{x}, \mathbf{z}_{\mathbf{1}}\right)=d_{\mathscr{H}}(\mathbf{y}, \mathbf{z}) .
\end{aligned}
$$

Proof. Since $\mathscr{H}(u)$ is the union of an increasing chain of finite Hamming spaces, every property of finite systems of points will hold in $\mathscr{H}(u)$ provided that the property does in the finite spaces $H_{m}$ defined by (1) beginning with some positive integer $m$. Therefore, it suffices to verify the existence and uniqueness of the point $\mathbf{z}_{1}$ in the hypotheses of the lemma for finite Hamming spaces. Take three points $\bar{x}, \bar{y}$, and $\bar{z}$ in $H_{m}$. To start with, suppose that $d_{H_{m}}(\bar{x}, \bar{y})=m$. Then $[\bar{x}, \bar{y}]=H_{m}$ and for $\bar{z}$ there exists a unique antipodal point $\bar{z}_{1}$, which means that $d_{H_{m}}\left(\bar{z}, \bar{z}_{1}\right)=m$. The point $\bar{z}_{1}$ satisfies (18). Suppose now that $d_{H_{m}}(\bar{x}, \bar{y})=k<m$. Then the vectors $\bar{x}, \bar{y}$, and $\bar{z}$ have $m-k$ common coordinates. Project these vectors onto the space $H_{k}$ so that $\bar{x}$ and $\bar{y}$ have no common coordinates. By the argument above, the point $\bar{t}$ antipodal to the projection of $\bar{z}$ onto $H_{k}$ satisfies the equalities of the form (18) and is uniquely determined by these conditions. Appending to $t$ the coordinates which are common with $\bar{x}, \bar{y}$, and $\bar{z}$ with the indices involved in the projection, we obtain a point $\bar{z}_{1} \in H_{m}$ satisfying (18). It is the only possible point by the uniqueness of the construction.

REMARK 1. If the hypotheses of the lemma are fulfilled for some points $\bar{x}, \bar{y}, \bar{z}$, and $\overline{z_{1}}$ of $H_{m}$ (or $\mathscr{H}(u)$ ) then we say that $\overline{z_{1}}$ is determined by the triple $[\bar{x}, \bar{y}, \bar{z}]$. Observe that if $\overline{z_{1}}$ is determined by $\bar{x}, \bar{y}$, and $\bar{z}$ then by the antipodality of the Hamming space $H_{m}$ the points $x, y$, and $z$ are determined by the triples $\left[\bar{z}, \overline{z_{1}}, \bar{y}\right],\left[\bar{z}, \overline{z_{1}}, \bar{x}\right]$, and $\left[\bar{x}, \bar{y}, \overline{z_{1}}\right]$ respectively.

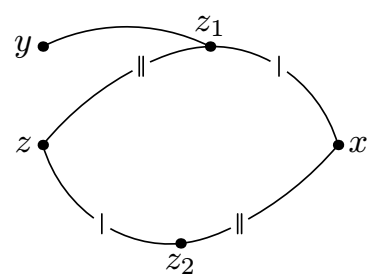

Fig. 2

Lemma 7. Given three points $\mathbf{x}, \mathbf{y}, \mathbf{z} \in \mathscr{H}(u)$ there exist unique points $\mathbf{z}_{\mathbf{1}}, \mathbf{z}_{\mathbf{2}} \in \mathscr{H}(u)$ with $\mathbf{z}_{\mathbf{1}}, \mathbf{z}_{\mathbf{2}} \in[\mathbf{x}, \mathbf{z}], \mathbf{z}_{\mathbf{1}} \in[\mathbf{x}, \mathbf{y}]$, and $\mathbf{x} \in\left[\mathbf{y}, \mathbf{z}_{\mathbf{2}}\right] ;$ furthermore, the point $\mathbf{z}_{\mathbf{2}}$ is determined by the triple $\left[\mathbf{x}, \mathbf{z}, \mathbf{z}_{\mathbf{1}}\right]$ (Fig. 2).

Proof. As in the proof of the previous lemma, it suffices to consider the case of finite Hamming spaces. Fix a positive integer $m$. Since the Hamming space $H_{m}$ is homogeneous, it suffices to prove the claim for the zero vector $\bar{x} \in H_{m}$. Writing $\bar{y}=\left(y_{1}, \ldots, y_{m}\right)$ and $\bar{z}=\left(z_{1}, \ldots, z_{m}\right)$, take the componentwise product $\bar{z}_{1}$ of $\bar{y}$ and $\bar{z}$. Then, obviously, $\bar{z}_{1} \in[\bar{x}, \bar{y}]$ and $\bar{z}_{1} \in[\bar{x}, \bar{z}]$; furthermore, $\bar{z}_{1}$ is uniquely determined by $\bar{y}$ and $\bar{z}$. Define $\bar{z}_{2}$ to be the point determined by the triple $\left[\bar{x}, \bar{z}, \overline{z_{1}}\right]$. Then by Remark $1 \bar{x}$ is determined by the triple $\left[\overline{z_{1}}, \overline{z_{2}}, \bar{z}\right]$, and so $\bar{x} \in\left[\overline{z_{1}}, \overline{z_{2}}\right]$. Since $\overline{z_{1}} \in[\bar{y}, \bar{x}]$, it follows that $\bar{x} \in\left[\bar{y}, \overline{z_{2}}\right]$, and all requirements of the lemma are fulfilled.

An isometry $g$ of a metric space $\left(Y, d_{Y}\right)$ is called an involution whenever $g^{2}=$ id and the distance $d_{Y}\left(x, x^{g}\right)$ is independent of the choice of $x$.

Each function $f \in \operatorname{Fun}_{C}\left(\partial T, Z_{2}\right)$ acts on the set $\Omega T_{\tau}$ of all clopen subsets of $\partial T_{\tau}$ as follows. The function $f$ determines the partition of $\partial T_{\tau}$ into the two subsets $O_{f}$ and $J_{f}$ :

$$
O_{f}=\left\{\gamma \in \partial T_{\tau} \mid f(\gamma)=0\right\}, \quad J_{f}=\left\{\gamma \in \partial T_{\tau} \mid f(\gamma)=1\right\} .
$$

The continuity of $f$ ensures that $O_{f}$ and $J_{f}$ are clopen. Given $A \in \Omega T_{\tau}$, put $A^{f}=A \triangle J_{f}$.

The mapping $w_{f}: A \rightarrow A^{f}, A \in \Omega T_{\tau}$, is well-defined since $A \triangle J_{f}$ is clopen. Moreover, it is a bijection onto $\Omega T_{\tau}$ and it preserves the metric $d_{\mu}$. Since

$$
\left(A^{f}\right)^{g}=\left(A \triangle J_{f}\right) \triangle J_{g}=A \triangle\left(J_{f} \triangle J_{g}\right)=A^{f g}
$$

for all $A \in \Omega T_{\tau}$ and $f, g \in \operatorname{Fun}_{C}\left(\partial T, Z_{2}\right)$, we obtain an action of $\operatorname{Fun}_{C}\left(\partial T, Z_{2}\right)$ on $\Omega T_{\tau}$. Since

$$
A^{f^{2}}=A \triangle\left(J_{f} \triangle J_{f}\right)=A
$$


for all $A \in \Omega T_{\tau}$ and $f \in \operatorname{Fun}_{C}\left(\partial T, Z_{2}\right)$, it follows that $f^{2}=$ id. Moreover, since

$$
A \triangle A^{f}=A \triangle\left(A \triangle J_{f}\right)=J_{f}
$$

for all $A \in \Omega T_{\tau}$ and $f \in \operatorname{Fun}_{C}\left(\partial T, Z_{2}\right)$, the distance $d_{\mu}\left(A, A^{f}\right)=\mu\left(A \triangle A^{f}\right)=\mu\left(J_{f}\right)$ is independent of the choice of $A$. Consequently, every element $f$ of $\operatorname{Fun}_{C}\left(\partial T, Z_{2}\right)$ acts on $\Omega T_{\tau}$ as an involution. Refer to these as the standard involutions of $\Omega T_{\tau}$.

Proof of Theorem 2. According to Theorem 1, to characterize the isometry group of $\mathscr{H}(u)$ is the same as to do the same with the isometry group of the space $\Omega T_{\tau}$ of clopen subsets of the boundary $\partial T_{\tau}$ of the spherically homogeneous tree $T_{\tau}$ equipped with the metric $d_{\mu}$ of $(10)$, where $\tau=\left(m_{1}, m_{2}, \ldots\right)$ is a strictly increasing divisible sequence of positive integers with $\operatorname{char}(\tau)=u$.

To start with, verify that every involution of $\Omega T_{\tau}$ is standard. Observe that the group of standard involutions acts on $\Omega T_{\tau}$ regularly, which means that given $A, B \in \Omega T_{\tau}$ there exists a unique standard involution $f$ with $A^{f}=B$. Therefore, it suffices to prove that given $A, B \in \Omega T_{\tau}$ there exists only one involution carrying $A$ to $B$. Take some involution $g$ of $\Omega T_{\tau}$ with $A^{g}=B$. Consider the images of other points $X$ of $\Omega T_{\tau}$. The following cases are possible:

(i) $X \in[A, B]$. In this case, since $g$ is an involution, we have

$$
\begin{aligned}
& d_{\mu}\left(A, X^{g}\right)=d_{\mu}\left(A^{g}, X\right)=d_{\mu}(B, X), \\
& d_{\mu}\left(B, X^{g}\right)=d_{\mu}\left(B^{g}, X\right)=d_{\mu}(A, X), \\
& d_{\mu}\left(X, X^{g}\right)=d_{\mu}\left(A, A^{g}\right)=d_{\mu}(A, B) .
\end{aligned}
$$

The first two equalities from (19) yield $X^{g} \in[A, B]$. By Lemma 6 this implies that there exists precisely one point with these properties, and so $X^{g}$ in this case is uniquely determined.

(ii) $A \in[X, B]$ (or $B \in[A, X]$ ). Since $g$ is an involution, we have

$$
d_{\mu}\left(X^{g}, X\right)=d_{\mu}(A, B), \quad d_{\mu}\left(X^{g}, A\right)=d_{\mu}(X, B), \quad d_{\mu}\left(X^{g}, B\right)=d_{\mu}(X, A) .
$$

By Lemma 6 in this case as well we find $X^{g}$ uniquely as the point determined by the triple $[X, B, A]$ (or $[X, B, A]$ ).

(iii) None of the points $X, A$, and $B$ belongs to the interval whose endpoints are the remaining two. In other words, (i) and (ii) are violated. Apply Lemma 7 to $A, B$, and $X$. By Lemma 7 there exist unique points $Z_{1}$ and $Z_{2}$ (see Fig. 2) such that $Z_{1}, Z_{2} \in[A, X], Z_{1} \in[A, B]$, and $X \in\left[B, Z_{2}\right]$; furthermore, $Z_{2}$ is determined by the triple $\left[A, X, Z_{1}\right]$. Then $X$ is determined by the triple $\left[Z_{1}, Z_{2}, A\right]$, and according to (i) and (ii) the points $Z_{1}^{g}$ and $Z_{2}^{g}$ are uniquely determined. In addition, since $g$ is an isometry, we have

$$
\begin{aligned}
& d_{\mu}\left(Z_{1}^{g}, X^{g}\right)=d_{\mu}\left(Z_{1}, X\right)=d_{\mu}\left(A, Z_{2}\right)=d_{\mu}\left(B, Z_{2}^{g}\right), \\
& d_{\mu}\left(Z_{2}^{g}, X^{g}\right)=d_{\mu}\left(Z_{2}, X\right)=d_{\mu}\left(A, Z_{1}\right)=d_{\mu}\left(B, Z_{1}^{g}\right), \\
& d_{\mu}\left(B, X^{g}\right)=d_{\mu}(A, X)=d_{\mu}\left(Z_{1}, Z_{2}\right)=d_{\mu}\left(Z_{1}^{g}, Z_{2}^{g}\right),
\end{aligned}
$$

which implies that $X^{g}$ is determined by the triple $\left[Z_{1}^{g}, Z_{2}^{g}, B\right]$, and so in this case the image $X^{g}$ is uniquely determined by the original points $X, A$, and $B$.

Thus, $g$ coincides with a standard involution, and since we chose it arbitrarily, every involution of $\Omega T_{\tau}$ is standard. Therefore, the involutions of $\Omega T_{\tau}$ constitute a normal subgroup of Iso $\Omega T_{\tau}$.

Consider an isometry $g$ of $\Omega T_{\tau}$ with $g(\varnothing)=A$. Then there exists a unique standard involution $h \in \operatorname{Fun}_{C}\left(\partial T, Z_{2}\right)$ with $g h^{-1}(\varnothing)=\varnothing$, that is, $g h^{-1}$ is included into the stabilizer $G_{0}$ of the point $\varnothing$ of $\Omega T_{\tau}$. Since $G_{0} \cap \operatorname{Fun}_{C}\left(\partial T, Z_{2}\right)=\mathrm{id}$, the isometry group of $\Omega T_{\tau}$ decomposes as the semidirect product

$$
\text { Iso } \Omega T_{\tau} \simeq \operatorname{Fun}_{C}\left(\partial T, Z_{2}\right) \rtimes G_{0}
$$

of the subgroup $G_{0}$ and the normal divisor $\operatorname{Fun}_{C}\left(\partial T, Z_{2}\right)$. 
Thus, it suffices to verify that $G_{0}$ coincides with $\operatorname{Aut}_{C}\left(\partial T_{\tau}, \mu\right)=\operatorname{Homeo}\left(\partial T_{\tau}\right) \cap \operatorname{Aut}\left(\partial T_{\tau}, \mu\right)$. This means that a transformation $g$ of $\partial T_{\tau}$ with $g(\varnothing)=\varnothing$ is an isometry of $\Omega T_{\tau}$ if and only if it is continuous and measure-preserving.

Suppose that $g$ is an isometry of $\Omega T_{\tau}$ with $g(\varnothing)=\varnothing$. Since both $g$ and $g^{-1}$ are isometries, they carry clopen subsets of $\partial T_{\tau}$ to clopen subsets, and so they are continuous. Therefore, $g \in \operatorname{Homeo}\left(\partial T_{\tau}\right)$. In addition, $g$ acts on $\partial T_{\tau}$ and carries measure zero sets to measure zero sets. This implies that the action of $g$ on $\partial T_{\tau}$ preserves the Bernoulli measure: $g \in \operatorname{Aut}\left(\partial T_{\tau}, \mu\right)$. Consequently, $G_{0} \subseteq \operatorname{Homeo}\left(\partial T_{\tau}\right) \cap \operatorname{Aut}\left(\partial T_{\tau}, \mu\right)$.

Suppose now that $g \in \operatorname{Homeo}\left(\partial T_{\tau}\right) \cap \operatorname{Aut}\left(\partial T_{\tau}, \mu\right)$. Since $g \in \operatorname{Homeo}\left(\partial T_{\tau}\right)$, it follows that both $g$ and $g^{-1}$ are continuous, and they carry clopen subsets of $\partial T_{\tau}$ to clopen subsets, thus determining bijective transformations of $\Omega T_{\tau}$. On the other hand, since $g \in \operatorname{Aut}\left(\partial T_{\tau}, \mu\right)$, it follows that $g$ preserves the measure of clopen subsets of $\partial T_{\tau}$ and carries measure zero sets to measure zero sets. Consequently, $g$ preserves the distance $d_{\mu}$ in $\Omega T_{\tau}$, and so it is an isometry of $\Omega T_{\tau}$. Thus, Aut ${ }_{C}\left(\partial T_{\tau}, \mu\right) \subseteq G_{0}$, and the isomorphism (17) holds.

The proof of the theorem is complete.

Since for every infinite strictly increasing divisible sequence $\tau$ the completion of the Hamming space of $\tau$-periodic sequences is isometric to the completion $\mathscr{C} \mathscr{H}$ of the space $\mathscr{H}$ of all periodic $(0,1)$-sequences, we can further characterize the isometry group of $\mathscr{C} \mathscr{H}$ as follows.

Corollary 3. The isometry group Iso $\mathscr{C} \mathscr{H}$ of the completion $\mathscr{C} \mathscr{H}$ of the space $\mathscr{H}$ of all periodic $(0,1)$-sequences is isomorphic as a transformation group to the measurable analog (15) of the hyperoctahedral group:

$$
\text { Iso } \mathscr{C} \mathscr{H} \simeq Z_{2} \imath_{\mu} \operatorname{Aut}(\partial T, \mu)
$$

where $T$ is some spherically homogeneous rooted tree whose spherical index is a strictly increasing sequence, while $\mu$ is the Bernoulli measure (9) on the $\sigma$-algebra of clopen sets of $\partial T$.

Proof. Observe firstly that, since $\mathscr{C} \mathscr{H}$ is the completion of $\mathscr{H}$, Lemmas 6 and 7 carry over to $\mathscr{C} \mathscr{H}$. In addition, by Proposition 1 the completion of $\mathscr{H}$ is isometric to the space $\Theta T$ of all measurable subsets (up to measure zero sets) of the space $(\partial T, \mu)$ equipped with the metric $d_{\mu}$ of (10). Arguing as in the proof of Theorem 2, we infer that the isometry group of $\Theta T$ decomposes as the semidirect product

$$
\text { Iso } \Theta T \simeq \operatorname{Fun}_{C}\left(\partial T, Z_{2}\right) \rtimes G_{0}
$$

of the subgroup $G_{0}$ (the stabilizer of $\varnothing$ ) and the normal divisor $\operatorname{Fun}_{C}\left(\partial T, Z_{2}\right)$. Therefore, it suffices to verify that a transformation $g$ of $\partial T$ with $g(\varnothing)=\varnothing$ is an isometry of $\Theta T$ if and only if it is measurepreserving. This follows directly from the definition of the metric $d_{\mu}$.

4.3. We can characterize the group of continuous measure-preserving automorphisms of the measure space $\left(\partial T_{\tau}, \mu\right)$, where $\tau=\left(m_{1}, m_{2}, \ldots\right)$ is a strictly increasing divisible sequence of positive integers, as follows. Take the sequence $S_{m_{1}}, S_{m_{2}}, \ldots$ of symmetric groups of degrees $m_{1}, m_{2}, \ldots$ Since $m_{i}$ equals the number of vertices on level $L_{i}$ of $T_{\tau}$, the group $S_{m_{i}}$ acts by permutations on $L_{i}$. Furthermore, the tree itself determines an embedding of $S_{m_{i}}$ into $S_{m_{i+1}}$, which is a strictly diagonal embedding in the sense of [10]; this means that on $L_{i+1}$ the image of $S_{m_{i}}$ acts naturally on each of its orbits.

Indeed, if $\pi \in S_{m_{i}}$ induces a permutation

$$
\hat{\pi}: v_{i} \rightarrow v_{\pi(i)}, \quad i=1,2, \ldots,
$$

of the vertices in $L_{i}$ then the corresponding permutation $\delta_{r_{i}}(\pi)$ of level $i+1$ vertices arises from the permutation of the rooted subtrees of $T_{\tau}$ with the roots in $L_{i}$ using $\hat{\pi}$. The mapping $\delta_{r_{i}}$ is an embedding of $S_{m_{i}}$ into $S_{m_{i+1}}$. This leads to the direct system $\left\langle S_{m_{i}}, \delta_{r_{i}}\right\rangle_{i \in \mathbb{N}}$ of symmetric groups, whose limit group

$$
S(\tau)=\lim _{\longrightarrow}\left\langle S_{m_{i}}, \delta r_{i}\right\rangle
$$


naturally acts on the boundary $\partial T_{\tau}$ of the tree. The group $S(\tau)$ belongs to the class of the so-called homogeneous symmetric groups [10] and is uniquely determined up to an isomorphism by the supernatural number $\operatorname{char}(\tau)$.

Equip the group of homeomorphisms $\operatorname{Homeo}\left(\partial T_{\tau}\right)$ with the standard metric

$$
\tilde{\rho}_{\tau}(g, f)=\max _{x \in \partial T_{\tau}} \rho_{\tau}\left(x^{g}, x^{f}\right)
$$

for all $g, f \in \operatorname{Homeo}\left(\partial T_{\tau}\right)$.

Lemma 8 [11]. Given a strictly increasing divisible sequence $\tau=\left(m_{1}, m_{2}, \ldots\right)$, the group $S(\tau)$ is an everywhere dense subgroup of $\operatorname{Aut}_{C}\left(\partial T_{\tau}\right)$ with the topology induced by the metric $\tilde{\rho}_{\tau}$.

On the other hand, acting on level $i$ vertices of $T_{\tau}$ by permutations, the symmetric group $S_{m_{i}}$ permutes subsets of vertices, that is, it acts on the points of the Hamming space $H_{m_{i}}$. Therefore, with this action it is a part of the isometry group of this space, which is the wreath product $Z_{2} \succ S_{m_{i}}$ acting on the cylindrical subsets of level $i$ of $\partial T_{\tau}$. Passing to level $i+1$, we obtain an embedding of $Z_{2} \succ S_{m_{i}}$ into $Z_{2} \imath S_{m_{i+1}}$ which embeds $S_{m_{i}}$ into $S_{m_{i+1}}$ as we described above, while the subgroup of involutions, isomorphic to $\underbrace{Z_{2} \times \cdots \times Z_{2}}_{m_{i}}$, embeds into the subgroup $\underbrace{Z_{2} \times \cdots \times Z_{2}}_{m_{i+1}}$ as

$$
\left(x_{1}, x_{2}, \ldots, x_{m_{i}}\right) \hookrightarrow(\underbrace{x_{1}, \ldots, x_{1}}_{r_{i}}, \underbrace{x_{2}, \ldots, x_{2}}_{r_{i}}, \ldots, \underbrace{x_{m_{i}}, \ldots, x_{m_{i}}}_{r_{i}}) .
$$

We can characterize the elements of the union of the increasing chain of subgroups $\underbrace{Z_{2} \times \cdots \times Z_{2}}_{m_{i}}$ as mappings from $\partial T_{\tau}$ into $Z_{2}$, for each of which there exists a partition of $\partial T_{\tau}$ into finitely many balls such that the mapping takes a constant value on each ball. Since $\partial T_{\tau}$ is a compact space, these mappings are continuous functions from $\partial T_{\tau}$ into $Z_{2}$, that is, they lie in $\operatorname{Fun}_{C}\left(\partial T, Z_{2}\right)$. Thus, the union of the increasing chain of subgroups $Z_{2} \curlywedge S_{m_{i}}$ is isomorphic to the semidirect product $\operatorname{Fun}_{C}\left(\partial T, Z_{2}\right) \rtimes S(\tau)$.

Similar to (20) equip $\operatorname{Fun}_{C}\left(\partial T_{\tau}, Z_{2}\right)$ with the metric

$$
\varrho_{\tau}(g, f)=\max _{x \in \partial T_{\tau}}\left(x^{g}+x^{f}\right),
$$

which induces the discrete topology on $\operatorname{Fun}_{C}\left(\partial T_{\tau}, Z_{2}\right)$. These arguments and Lemma 8 imply

Theorem 3. Given a strictly increasing divisible sequence $\tau=\left(m_{1}, m_{2}, \ldots\right)$ of positive integers, the isometry group Iso $\mathscr{H}(\tau)$ of the Hamming space of $\tau$-periodic sequences is the closure of the inductive limit of isometry groups Iso $\mathscr{H}_{m_{i}}$ of finite Hamming spaces regarded as a subgroup of $\operatorname{Fun}_{C}\left(\partial T_{\tau}, Z_{2}\right) \rtimes$ Homeo $\left(\partial T_{\tau}\right)$ in the Tychonoff product of topologies induced by the metrics $\varrho_{\tau}$ and $\tilde{\rho}_{\tau}$.

PROOF. It suffices to show that for every isometry $f \in \operatorname{Iso} \mathscr{H}(\tau)$ there exists a sequence $\left\{f_{n}, n \geq 1\right\}$ of elements of the inductive limit of isometry groups of finite Hamming spaces such that $\lim _{n \rightarrow \infty} f_{n}=f$ in the $\operatorname{space} \operatorname{Fun}_{C}\left(\partial T_{\tau}, Z_{2}\right) \rtimes \operatorname{Homeo}\left(\partial T_{\tau}\right)$. Since the group $\operatorname{Fun}_{C}\left(\partial T_{\tau}, Z_{2}\right) \rtimes \operatorname{Homeo}\left(\partial T_{\tau}\right)$ is a semidirect product, we can express every element of the isometry group Iso $\mathscr{H}(\tau)$ of the Hamming space of $\tau$-periodic sequences as a pair of elements of $\operatorname{Fun}_{C}\left(\partial T_{\tau}, Z_{2}\right)$ and Homeo $\left(\partial T_{\tau}\right)$, and, moreover, considering Theorem 2, of $\operatorname{Fun}_{C}\left(\partial T_{\tau}, Z_{2}\right)$ and $\operatorname{Homeo}\left(\partial T_{\tau}\right) \cap \operatorname{Aut}\left(\partial T_{\tau}, \mu\right)$. Consequently, each isometry uniquely decomposes as $f=(g, h)$ with $g \in \operatorname{Fun}_{C}\left(\partial T_{\tau}, Z_{2}\right)$ and $h \in \operatorname{Homeo}\left(\partial T_{\tau}\right) \cap \operatorname{Aut}\left(\partial T_{\tau}, \mu\right)$. Then the existence of a sequence $\left\{f_{n}, n \geq 1\right\}$ is equivalent to the existence of two sequences $\left\{g_{n}, n \geq 1\right\}$ and $\left\{h_{n}, n \geq 1\right\}$ in $\operatorname{Fun}_{C}\left(\partial T_{\tau}, Z_{2}\right)$ and Homeo $\left(\partial T_{\tau}\right) \cap \operatorname{Aut}\left(\partial T_{\tau}, \mu\right)$ respectively with $\lim _{n \rightarrow \infty} g_{n}=g$ and $\lim _{n \rightarrow \infty} h_{n}=h$. The arguments made prior to the theorem imply that we can characterize the inductive limit of isometry groups of finite Hamming spaces as the semidirect product of the groups $\operatorname{Fun}_{C}\left(\partial T, Z_{2}\right)$ and $S(\tau)$. Therefore, the existence of $\left\{g_{n}, n \geq 1\right\}$ is obvious, while the existence of $\left\{h_{n}, n \geq 1\right\}$ follows from Lemma 8 . 


\section{References}

1. Blanchard F., Formenti E., and Kurka P., "Cellular automata in Cantor, Besicovitch and Weil topological spaces," Complex Syst., 11, 107-123 (1997).

2. Vershik A. M., "Theory of decreasing sequences of measurable partitions," St. Petersburg Math. J., 6, No. 4, 705-761 (1995).

3. Vershik A. M., "Decreasing sequences of measurable partitions and their applications," Dokl. Akad. Nauk SSSR, 193, No. 4, 748-751 (1970).

4. Vershik A. M., "The Kantorovich metric: initial history and little-known applications," J. Math. Sci. (N.Y.), 133, No. 4, 1410-1417 (2006).

5. Guseva O. V., "Classification of the sequences of measurable partitions," Vestnik LGU, 1, No. 1, 14-23 (1965).

6. Rubshteı̆n B. A., "Decreasing sequences of measurable partitions," Dokl. Akad. Nauk SSSR, 205, No. 3, 526-530 (1972).

7. Cameron P. J. and Tarzi S., "Limits of cubes," Topology Appl., 155, 1454-1461 (2008).

8. Grigorchuk R. I., Nekrashevych V. V., and Sushchanskiı̌ V. I., "Automata, dynamical systems, and groups," Proc. Steklov Inst. Math., 231, 128-203 (2000).

9. Kaloujnine L. A., Sushchanskiu V. I., and Ustimenko-Bakumovskiŭ V. A., "Exponentiation in permutation group theory and its applications," in: Mat. VI All-Union Conf. on Group Theory, Inst. Mat. AN UkrainSSR, Kiev, 1979, pp. 135-145.

10. Kroshko N. V. and Sushchansky V. I., "Direct limits of symmetric and alternating groups with strictly diagonal embeddings," Arch. Math., 71, No. 3, 173-182 (1998).

11. Lavrenyuk Ya. V. and Nekrashevych V. V., "Groups of measure-preserving homeomorphisms of the Cantor set," Dop. Akad. Nauk Ukrain., No. 6, 28-31 (2008).

B. V. OLIYNYK

Taras Shevchenko Kiev National University, Kiev, Ukraine

E-mail address: bogdana.oliynyk@gmail.com

V. I. SUSHCHANSKII

Silesian University of Technology, Gliwice, Poland

E-mail address: vitaliy.sushchanskyy@polsl.pl 